Real Analysis Exchange

Vol. 23(2), 1997-1998, pp. 545-552

Antonis Bisbas, University of the Aegean, Karlovasi 83200, Samos, Greece

e-mail: Bisbas@kerkis.math.aegean.gr

\title{
A NOTE ON THE DISTRIBUTION OF DIGITS IN TRIADIC EXPANSIONS
}

\begin{abstract}
We estimate the Hausdorff dimension of some Borel sets determined by the digits in triadic expansions.
\end{abstract}

\section{Introduction}

Let $x=\sum_{n=1}^{\infty} \frac{\varepsilon_{n}(x)}{3^{n}}$, where $\varepsilon_{n}(x) \in\{0,1,2\}$, be the 3 -adic expansion of $x \in[0,1]$. Our purpose is to estimate the Hausdorff dimension of the set

$$
M_{k, m}(q)=\left\{x: \lim _{N \rightarrow \infty} \frac{1}{N} \sum_{n=1}^{N} \varepsilon_{n}^{k}(x) \varepsilon_{n+1}^{m}(x)=q\right\}
$$

where $k, m \in\{0,1,2\}, k+m \geq 1$ and $q \in\left[0,2^{k+m}\right]$. The proof is based on the construction of a suitable measure. It would be desirable to see the analogous problem for $x$ expressed as a decimal in the scale $r=4,5, \ldots$ but we have not been able to do this; for $r=2$ see [3], extended in [4]. Similar results have been obtained in [1], [2], [5], [7], [11], [13]. In section 3 we give a multifractal analysis of some measures related to this work.

Let $P=\left(p_{i j}\right), i, j=0,1,2$, be a stochastic irreducible matrix, $P^{(0)}=$ $\left(\pi_{0}, \pi_{1}, \pi_{2}\right)$ be a probability vector such that $P^{(0)} P=P^{(0)}$ and $E_{N}(x)$ be the interval of the form $\left[\frac{\kappa}{3^{N}}, \frac{\kappa+1}{3^{N}}\right)$ containing $x, \kappa=0,1, \ldots, 3^{N}-1$. We define the measure $\mu$ by its values on $E_{N}(x)$;

$$
\mu\left(E_{N}(x)\right)=\pi_{\varepsilon_{1}(x)} \prod_{n=1}^{N-1} p_{\varepsilon_{n}(x) \varepsilon_{n+1}(x)} .
$$

Key Words: Hausdorff dimension, multifractal

Mathematical Reviews subject classification: Primary: 28A78

Received by the editors July 18, 1997 
It is well known [2] that if

$$
M=\left\{x: \lim _{N \rightarrow \infty} \frac{\log \mu\left(E_{N}(x)\right)}{-N \log 3}=\delta_{P}\right\}
$$

where

$$
\delta_{P}=\frac{-1}{\log 3} \sum_{i, j=0}^{2} \pi_{i} p_{i j} \log p_{i j},
$$

then $\mu(M)=1$ and the Hausdorff dimension of $M, \operatorname{dim} M$, is $\delta_{P}$.

\section{The Hausdorff Dimension of $M_{k, m}(q)$}

In this section it is show that we can choose $P$ in such a way that $M=$ $M_{k, m}(q)$. From (2) we obtain

$$
\log \mu\left(E_{N}(x)\right)=\log \pi_{\varepsilon_{1}(x)}+\sum_{n=1}^{N-1} \sum_{i, j=0}^{2} \delta_{\varepsilon_{n}(x), i} \delta_{\varepsilon_{n+1}(x), j} \log p_{i j},
$$

where $\delta_{\text {., }}$ is the usual Kronecker symbol. We observe that

$$
\delta_{\varepsilon_{n}(x), i}=(-1)^{i} \frac{\prod_{\substack{j=0 \\ j \neq i}}^{2}\left(j-\varepsilon_{n}(x)\right)}{i !(2-i) !}=\sum_{k=0}^{2} c_{k i} \varepsilon_{n}^{k}(x), \quad c_{k i} \in \mathbb{R} .
$$

Let $\log A_{k m}=\sum_{i, j=0}^{2} c_{k i} c_{m j} \log p_{i j}$. Then

$$
\log \mu\left(E_{N}(x)\right)=\log \pi_{\varepsilon_{1}(x)}+\sum_{n=1}^{N-1} \sum_{k, m=0}^{2} \varepsilon_{n}^{k}(x) \varepsilon_{n+1}^{m}(x) \log A_{k m}
$$

and

$$
\begin{aligned}
\lim _{N \rightarrow \infty} \frac{1}{N} \log \mu\left(E_{N}(x)\right) & =\lim _{N \rightarrow \infty} \frac{1}{N} \sum_{n=1}^{N}\left[\log A_{00}+\varepsilon_{n}(x) \log \left(A_{10} A_{01}\right)\right. \\
& \left.+\varepsilon_{n}^{2}(x) \log \left(A_{20} A_{02}\right)+\sum_{k, m=1}^{2} \varepsilon_{n}^{k}(x) \varepsilon_{n+1}^{m}(x) \log A_{k m}\right]
\end{aligned}
$$

Set $E_{10}=E_{01}=A_{10} A_{01}, E_{20}=E_{02}=A_{20} A_{02}$ and $E_{k m}=A_{k m}$ for $k m \neq 0$. We will make the following assumptions: if for some $E_{i j}$ the product $i j$ is 0 then $j=0$ and if for some $M_{k, m}$ the product $k m$ is 0 then $m=0$. 
Theorem. Theorem Let $M_{k, m}(q)$ be as in (1). Then $\operatorname{dim} M_{k, m}(q)=\delta_{P}$, where $P=\left(p_{i j}\right), i, j=0,1,2$, is a solution of the system

$$
E_{i j}=1, \quad(i, j) \neq(k, m), \quad q=t_{k m}=\sum_{i, j=0}^{2} i^{k} j^{m} \pi_{i} p_{i j}
$$

Note. We adopt the convention that $0^{0}=1$.

Proof. Suppose the stochastic irreducible matrix $P=\left(p_{i j}\right)$ is a solution of the above system. Then from (3) and (5) it follows that

$$
M=\left\{x: \lim _{N \rightarrow \infty} \frac{-1}{N \log 3} \sum_{n=1}^{N}\left[\log A_{00}+\varepsilon_{n}^{k}(x) \varepsilon_{n+1}^{m}(x) \log E_{k m}\right]=\delta_{P}\right\} .
$$

Since $\int \varepsilon_{1}^{k}(x) \varepsilon_{2}^{m}(x) d \mu(x)=t_{k m}$, the ergodic theorem [2] shows that

$$
M=M_{k, m}\left(t_{k m}\right)=M_{k, m}(q)
$$

which is the desired conclusion. We need only show the existence of a solution to the system. For simplicity of notation we write $x_{i}=p_{i 0}, y_{i}=p_{i 1}, i=0,1,2$. By (4) it is obvious that

$$
\begin{aligned}
& c_{00}=1, c_{10}=-\frac{3}{2}, c_{20}=\frac{1}{2}, c_{01}=0, c_{11}=2, c_{21}=-1, \\
& c_{02}=0, c_{12}=-\frac{1}{2}, c_{22}=\frac{1}{2} .
\end{aligned}
$$

Hence in any case (with respect to $k, m$ ) we have five of the following six relations, $(a)-(f)$, and the relation $(g)$ :

$$
\begin{aligned}
E_{10}= & x_{0}^{-3} y_{0}^{2}\left(1-x_{0}-y_{0}\right)^{-\frac{1}{2}} x_{1}^{2} x_{2}^{-\frac{1}{2}}=1 \\
E_{20}= & x_{0} y_{0}^{-1}\left(1-x_{0}-y_{0}\right)^{\frac{1}{2}} x_{1}^{-1} x_{2}^{\frac{1}{2}}=1 \\
E_{11}= & x_{0}^{\frac{9}{4}} y_{0}^{-3}\left(1-x_{0}-y_{0}\right)^{\frac{3}{4}} x_{1}^{-3} y_{1}^{4}\left(1-x_{1}-y_{1}\right)^{-1} \\
& \times x_{2}^{\frac{3}{4}} y_{2}^{-1}\left(1-x_{2}-y_{2}\right)^{\frac{1}{4}}=1 \\
E_{21}= & x_{0}^{-\frac{3}{4}} y_{0}\left(1-x_{0}-y_{0}\right)^{-\frac{1}{4}} x_{1}^{\frac{3}{2}} y_{1}^{-2}\left(1-x_{1}-y_{1}\right)^{\frac{1}{2}} \\
& \times x_{2}^{-\frac{3}{4}} y_{2}\left(1-x_{2}-y_{2}\right)^{-\frac{1}{4}}=1 \\
E_{12}= & x_{0}^{-\frac{3}{4}} y_{0}^{\frac{3}{2}}\left(1-x_{0}-y_{0}\right)^{-\frac{3}{4}} x_{1} y_{1}^{-2}\left(1-x_{1}-y_{1}\right)
\end{aligned}
$$




$$
\begin{aligned}
(f) \quad E_{22}= & x_{0}^{\frac{1}{4}} y_{0}^{-\frac{1}{2}}\left(1-x_{0}-y_{0}\right)^{\frac{1}{4}} x_{1}^{-\frac{1}{2}} y_{1}\left(1-x_{1}-y_{1}\right)^{-\frac{1}{2}} \\
& \times x_{2}^{\frac{1}{4}} y_{2}^{-\frac{1}{2}}\left(1-x_{2}-y_{2}\right)^{\frac{1}{4}}=1 \\
\text { (g) } \quad & t_{k m}
\end{aligned}
$$

We give the proof only for the case $k=m=1$; the other cases may be proved in much the same way. For the convenience of the reader we write the equations which we have in any case.

(i) $\mathbf{k}=\mathbf{m}=\mathbf{1}$. We have the equations $(a),(b),(d),(e),(f)$ and $(g)$. An easy computation shows that we have $(g)$ and

$$
\begin{aligned}
\frac{x_{0}\left(1-x_{0}-y_{0}\right)}{y_{0}^{2}} & =\frac{x_{1}\left(1-x_{1}-y_{1}\right)}{y_{1}^{2}}=\frac{x_{2}\left(1-x_{2}-y_{2}\right)}{y_{2}^{2}} \\
& =\frac{x_{1}^{2}}{x_{0} x_{2}}=\frac{y_{1}^{2}}{y_{0} y_{2}}, \quad x_{0}^{2}=y_{0} x_{1} .
\end{aligned}
$$

Combining these we obtain

$$
\begin{aligned}
x_{1} & =\frac{x_{0}^{2}}{y_{0}}, x_{2}=\frac{x_{0}^{2}}{1-x_{0}-y_{0}}, \\
F_{1}\left(x_{0}, y_{0}, y_{1}\right) & =y_{1}^{2}+y_{1} \frac{x_{0} y_{0}}{1-x_{0}-y_{0}}-\frac{x_{0} y_{0}}{1-x_{0}-y_{0}}\left(1-\frac{x_{0}^{2}}{y_{0}}\right)=0, \\
F_{2}\left(x_{0}, y_{0}, y_{1}\right) & =y_{1}^{4}+y_{1}^{2} \frac{x_{0}^{2} y_{0}}{1-x_{0}-y_{0}}-x_{0}^{3}\left(1-\frac{x_{0}^{2}}{1-x_{0}-y_{0}}\right)=0, \\
y_{2} & =\frac{y_{1}^{2} y_{0}}{x_{0}\left(1-x_{0}-y_{0}\right)} .
\end{aligned}
$$

From (6) we see that $x_{0}, y_{0}$ must be such that $x_{0}^{2}<y_{0}<1-x_{0}-x_{0}^{2}$ (and so $\left.x_{0} \in\left(0, \frac{1}{2}\right)\right)$. Let $\mathbf{x}_{\mathbf{0}} \in\left(0, \frac{1}{2}\right)$ and $y_{0} \in\left(\mathbf{x}_{\mathbf{0}}^{\mathbf{2}}, 1-\mathbf{x}_{\mathbf{0}}-\mathbf{x}_{\mathbf{0}}^{\mathbf{2}}\right)$. The equation (7) has a unique positive solution $y_{1}^{\prime}=h^{\prime}\left(\mathbf{x}_{\mathbf{0}}, y_{0}\right)$. The same holds for (8) with $y_{1}^{\prime \prime}=h^{\prime \prime}\left(\mathbf{x}_{\mathbf{0}}, y_{0}\right)$. Then for $y_{1}^{\prime}=y_{1}^{\prime \prime}$ we must have

$$
H\left(\mathbf{x}_{\mathbf{0}}, y_{0}\right)={h^{\prime}}^{2}\left(\mathbf{x}_{\mathbf{0}}, y_{0}\right)-h^{\prime \prime}\left(\mathbf{x}_{\mathbf{0}}, y_{0}\right)=0 .
$$

Since $H\left(\mathbf{x}_{\mathbf{0}}, \mathbf{x}_{\mathbf{0}}^{\mathbf{2}}\right)<0$ and $H\left(\mathbf{x}_{\mathbf{0}}, 1-\mathbf{x}_{\mathbf{0}}-\mathbf{x}_{\mathbf{0}}^{\mathbf{2}}\right)>0$, there exists $\mathbf{y}_{\mathbf{0}} \in\left(\mathbf{x}_{\mathbf{0}}^{\mathbf{2}}, 1-\mathbf{x}_{\mathbf{0}}-\right.$ $\left.\mathbf{x}_{\mathbf{0}}^{\mathbf{2}}\right)$, such that $H\left(\mathbf{x}_{\mathbf{0}}, \mathbf{y}_{\mathbf{0}}\right)=0$. Hence for this $\left(\mathbf{x}_{\mathbf{0}}, \mathbf{y}_{\mathbf{0}}\right)$ we have $y_{1}^{\prime}=y_{1}^{\prime \prime}=\mathbf{y}_{\mathbf{1}}$. It is easy to check that $\mathbf{y}_{\mathbf{1}} \in\left(0,1-\frac{\mathbf{x}_{0}^{2}}{\mathbf{y}_{0}}\right)$ and $y_{2} \in\left(0,1-\frac{\mathbf{x}_{0}^{2}}{1-\mathbf{x}_{\mathbf{0}}-\mathbf{y}_{\mathbf{0}}}\right)$. The Jacobian $\frac{\partial\left(F_{1}, F_{2}\right)}{\partial\left(y_{0}, y_{1}\right)}\left(\mathbf{x}_{\mathbf{0}}, \mathbf{y}_{\mathbf{0}}, \mathbf{y}_{\mathbf{1}}\right)$ is positive $\left(\partial F_{1} / \partial y_{0}\left(\mathbf{x}_{\mathbf{0}}, \mathbf{y}_{\mathbf{0}}, \mathbf{y}_{\mathbf{1}}\right)<0\right)$ and so the 
implicit function theorem [10, p. 168] gives that there is a neighborhood $B$ of $\mathbf{x}_{\mathbf{0}}$ and uniquely determined continuous functions $g_{0}, g_{1}$, defined on $B$ such that $y_{0}=g_{0}\left(x_{0}\right), y_{1}=g_{1}\left(x_{0}\right)$ and

$$
F_{1}\left(x_{0}, g_{0}\left(x_{0}\right), g_{1}\left(x_{0}\right)\right)=F_{2}\left(x_{0}, g_{0}\left(x_{0}\right), g_{1}\left(x_{0}\right)\right)=0 .
$$

Since the Jacobian is positive everywhere in our domain, we can have the functions $g_{0}, g_{1}$ defined on $\left(0, \frac{1}{2}\right)$ and satisfy (10). Thus by (6) and (9) we get continuous functions $f_{i}, g_{2}$ such that $x_{i}=f_{i}\left(x_{0}\right), y_{2}=g_{2}\left(x_{0}\right), x_{0} \in\left(0, \frac{1}{2}\right)$. From this we conclude that $t_{11}$ is also a continuous function of $x_{0}$. It is obvious that

$$
y_{0}=g_{0}\left(x_{0}\right) \rightarrow \frac{1}{4}, x_{i}=f_{i}\left(x_{0}\right) \rightarrow 1, y_{i}=g_{i}\left(x_{0}\right) \rightarrow 0, i=1,2 \text { as } x_{0} \rightarrow \frac{1}{2},
$$

which gives $t_{11} \rightarrow 0$, as $x_{0} \rightarrow \frac{1}{2}$. If $x_{0}$ is near to 0 , then $H\left(x_{0}, x_{0}^{\frac{1}{4}}\right)>$ $0, H\left(x_{0}, x_{0}\right)<0$, and $F_{2}\left(x_{0}, y_{0}, \frac{\sqrt{x_{0}^{\frac{3}{2}}}}{y_{0}}\right)>0$. Therefore,

$$
g_{i}\left(x_{0}\right) \rightarrow 0, f_{i}\left(x_{0}\right) \rightarrow 0, i=0,1,2, \text { as } x_{0} \rightarrow 0
$$

and finally that $t_{11} \rightarrow 4$ as $x_{0} \rightarrow 0$. Let $q \in(0,4)$. By the above there is $x_{0} \in\left(0, \frac{1}{2}\right)$ and so a stochastic irreducible matrix $P$, such that $M=M_{1,1}(q)$ which is our assertion.

If $q=4$, then by [7] we have $\operatorname{dim} M_{1,1}(4)=0$. We can extend the proof to assume $P$ such that $x_{i}=0, y_{i}=0$ and $\delta_{P}=0$ ( $P$ is not irreducible).

If $q=0$, then for $P$ such that $x_{0}=\frac{1}{2}, y_{0}=\frac{1}{4}, x_{i}=1, y_{i}=0, i=1,2$ we take that $M$ is a proper subset of $M_{1,1}(0)$ and so $\operatorname{dim} M_{1,1}(0) \geq \delta_{P}$. We apply another version of (3) (see [2, p. 144]) and use the results for $q \in(0,4)$ to obtain $\operatorname{dim} M_{1,1}(0)=\delta_{P}$.

(ii) $\mathbf{k}=\mathbf{2}, \mathbf{m}=\mathbf{1}$. We have the equations $(a),(b),(c),(e),(f)$ and $(g)$ or equivalently $(g)$ and

$$
\begin{aligned}
\frac{x_{0}\left(1-x_{0}-y_{0}\right)}{y_{0}^{2}} & =\frac{x_{1}\left(1-x_{1}-y_{1}\right)}{y_{1}^{2}}=\frac{x_{2}\left(1-x_{2}-y_{2}\right)}{y_{2}^{2}} \\
& =\frac{x_{1}^{2}}{x_{0} x_{2}}, \frac{x_{1}^{4}}{x_{0}^{3} x_{2}}=\frac{y_{1}^{4}}{y_{0}^{3} y_{2}}, \quad x_{0}^{2}=y_{0} x_{1} .
\end{aligned}
$$

As in case (i), we obtain (6), (7) and

$$
\begin{aligned}
F_{3}\left(x_{0}, y_{0}, y_{1}\right) & =y_{1}^{8}+y_{1}^{4} \frac{x_{0}^{4} y_{0}}{1-x_{0}-y_{0}}-x_{0}^{7}\left(1-\frac{x_{0}^{2}}{1-x_{0}-y_{0}}\right)=0, \\
y_{2} & =\frac{y_{1}^{4} y_{0}}{x_{0}^{3}\left(1-x_{0}-y_{0}\right)} .
\end{aligned}
$$


We can now proceed analogously to the proof of case (i).

(iii) $\mathbf{k}=\mathbf{1}, \mathbf{m}=\mathbf{2}$. We have the equations $(a),(b),(c),(d),(f)$ and $(g)$ or equivalently $(g)$ and

$$
\begin{aligned}
\frac{x_{0}^{3}\left(1-x_{0}-y_{0}\right)}{y_{0}^{4}} & =\frac{x_{1}^{3}\left(1-x_{1}-y_{1}\right)}{y_{1}^{4}}=\frac{x_{2}^{3}\left(1-x_{2}-y_{2}\right)}{y_{2}^{4}} \\
& =\frac{x_{1}^{4}}{x_{0}^{3} x_{2}}, \frac{x_{1}^{2}}{x_{0} x_{2}}=\frac{y_{1}^{2}}{y_{0} y_{2}}, \quad x_{0}^{2}=y_{0} x_{1} .
\end{aligned}
$$

A simple computation gives $(g),(6),(9)$ and

$$
\begin{aligned}
& F_{4}\left(x_{0}, y_{0}, y_{1}\right)=y_{1}^{4}+y_{1} \frac{x_{0}^{3} y_{0}}{1-x_{0}-y_{0}}-\frac{x_{0}^{3} y_{0}}{1-x_{0}-y_{0}}\left(1-\frac{x_{0}^{2}}{y_{0}}\right)=0, \\
& F_{5}\left(x_{0}, y_{0}, y_{1}\right)=y_{1}^{8}+y_{1}^{2} \frac{x_{0}^{6} y_{0}}{1-x_{0}-y_{0}}-x_{0}^{7}\left(1-\frac{x_{0}^{2}}{1-x_{0}-y_{0}}\right)=0 .
\end{aligned}
$$

The equation (12) has a unique positive solution $y_{1}^{\prime}=h^{\prime}\left(x_{0}, y_{0}\right)$. The same holds for (13) with $y_{1}^{\prime \prime}=h^{\prime \prime}\left(x_{0}, y_{0}\right)$. We must have $H\left(x_{0}, y_{0}\right)={h^{\prime}}^{2}\left(x_{0}, y_{0}\right)-$ $h^{\prime \prime}\left(x_{0}, y_{0}\right)=0$. The rest of the proof runs as in case (i).

(iv) $\mathbf{k}=\mathbf{2}, \mathbf{m}=\mathbf{2}$. We have the equations $(a),(b),(c),(d),(e)$ and $(g)$ or equivalent $(g)$ and

$$
\begin{aligned}
\frac{x_{0}^{3}\left(1-x_{0}-y_{0}\right)}{y_{0}^{4}} & =\frac{x_{1}^{3}\left(1-x_{1}-y_{1}\right)}{y_{1}^{4}}=\frac{x_{2}^{3}\left(1-x_{2}-y_{2}\right)}{y_{2}^{4}} \\
& =\frac{x_{1}^{4}}{x_{0}^{3} x_{2}}=\frac{y_{1}^{4}}{y_{0}^{3} y_{2}}, x_{0}^{2}=y_{0} x_{1} .
\end{aligned}
$$

As in previous cases we obtain (6), (11), (12) and

$$
F_{6}\left(x_{0}, y_{0}, y_{1}\right)=y_{1}^{16}+y_{1}^{4} \frac{x_{0}^{12} y_{0}}{1-x_{0}-y_{0}}-x_{0}^{15}\left(1-\frac{x_{0}^{2}}{1-x_{0}-y_{0}}\right)=0 .
$$

The rest of the proof is similar to that in case (iii).

$(\mathbf{v}) \mathbf{k}=\mathbf{2}, \mathbf{m}=\mathbf{0}$. We have the equations $(a),(c),(d),(e),(f)$ and $(g)$ or equivalent $(g)$ and

$$
\begin{gathered}
\frac{x_{0}\left(1-x_{0}-y_{0}\right)}{y_{0}^{2}}=\frac{x_{1}\left(1-x_{1}-y_{1}\right)}{y_{1}^{2}}=\frac{x_{2}\left(1-x_{2}-y_{2}\right)}{y_{2}^{2}}=\frac{x_{1}^{4} y_{0}^{2}}{x_{2} x_{0}^{5}}, \\
\frac{x_{1}^{2}}{x_{0} x_{2}}=\frac{y_{1}^{2}}{y_{0} y_{2}}, \quad \frac{x_{1}^{4}}{x_{0}^{3} x_{2}}=\frac{y_{1}^{4}}{y_{0}^{3} y_{2}} .
\end{gathered}
$$


We see at once that

$$
\begin{gathered}
x_{0}=x_{1}=x_{2}, y_{0}=y_{1}=y_{2}, \\
y_{0}^{4}-x_{0}^{3}\left(1-x_{0}-y_{0}\right)=0 .
\end{gathered}
$$

The proof is immediate.

(vi) $\mathbf{k}=\mathbf{1}, \mathbf{m}=\mathbf{0}$. We have the equations $(b)-(f)$ and $(g)$. The result is well known, see [7, pp. 77].

Corollary. Under the hypotheses of Theorem we have

$$
\operatorname{dim} M_{k, m}(q)=\frac{-1}{\log 3}\left[\log x_{0}+q \log \frac{g_{1}\left(x_{0}\right)}{x_{0}}\right] .
$$

Proof. By $(a)-(f)$ we get $\log E_{k m}=\log \frac{y_{1}}{x_{0}}=\log \frac{g_{1}\left(x_{0}\right)}{x_{0}}$. Since $\log A_{00}=$ $\log x_{0}$ we obtain $\delta_{P}=\frac{-1}{\log 3}\left[\log x_{0}+q \log \frac{g_{1}\left(x_{0}\right)}{x_{0}}\right]$, which completes the proof.

\section{A Multifractal Analysis}

The multifractal analysis of a Borel probability measure $\nu$ on $[0,1],[6],[8]$, [9], [12], is the study of the Hausdorff dimension of the sets

$$
E_{c}=\left\{x: \lim _{N \rightarrow \infty} \frac{\log \nu\left(E_{N}(x)\right)}{-N \log 3}=c\right\}, \quad c \in \mathbb{R} .
$$

Proposition. Let $\mu$ be a measure as in $(2)$, where $P=\left(p_{i j}\right)$ is such that $E_{i j}=1$, for $(i, j) \neq(k, m), k, m \in\{0,1,2\}, k+m \geq 1$. Then

$$
\operatorname{dim} E_{c}=\operatorname{dim} M_{k, m}(q),
$$

where $q=\frac{c \log 3+\log p_{00}}{\log \left(p_{00} p_{11}^{-1}\right)}$.

Proof. By assumption and (5) it follows that

$$
E_{c}=\left\{x: \lim _{N \rightarrow \infty} \frac{-1}{N \log 3} \sum_{n=1}^{N}\left[A_{00}+\varepsilon_{n}^{k}(x) \varepsilon_{n+1}^{m}(x) \log E_{k m}\right]=c\right\},
$$

$\log E_{k m}=\log \left(p_{00}^{-1} p_{11}\right)$ and $A_{00}=\log p_{00}$. The proof is straightforward. It is clear that $c$ must be such that $0 \leq \frac{c \log 3+\log p_{00}}{\log \left(p_{00} p_{11}^{-1}\right)} \leq 2^{k+m}$, otherwise the set $E_{c}$ is empty. If $\log \left(p_{00} p_{11}^{-1}\right)=0$, then our measure is that of Lebesque as is easy to check. 


\section{References}

[1] A. S. Besicovitch, On the sum of digits of real numbers represented in the dyadic system, Math. Annalen 110 (1934), 321-330.

[2] P. Billingsley, Ergodic theory and information, New York: Willey 1965.

[3] A. Bisbas, A note on the distribution of digits in dyadic expansions, C. R. Acad. Sci Paris 318 (1994), 105-109.

[4] A. Bisbas, C. Karanikas and G. Proios, On the distribution of digits in dyadic expansions, Results in Mathematics, 33 (1998), 40-49.

[5] H. Cajar, Billingsley Dimension in Propability Spaces, Lect. Notes Math., vol. 892.

[6] R. Cawley and R. D. Mauldin, Multifractal decompositions of Moran fractals, Advances in Mathematics 92 (1992), 196-236.

[7] H. G. Eggleston, Sets of fractional dimensions which occur in some problems of number theory, Pr. London Math. Soc. (2), 54 (1952), 42-93.

[8] A. H. Fan, Sur les dimensions de mesures, Studia Math. 111 (1994), 1-17.

[9] J. P. Kahane, Fractals and random measures, Bull. Sc. Math. 2o serie 117 (1993), 153-159.

[10] H. L. Loomis and S. Sternberg, Advanced calculus, Addison-Wesley, 1968.

[11] R. D. Mauldin and S. C. Williams, Hausdorff dimension in graph directed constructions, Trans. Am. Math. Soc. 309 (1988), 811-829.

[12] L. Olsen, A multifractal formalism, Advances in Math. 116 (1995), 82-195.

[13] B. Volkmann, Über Hausdorffsche Dimensionen von Mengen, die durch Zifferneigenschaften charakterisiert sind I; Math. Zeitschr. 58 (1953), $284-287$. 